\title{
Berberine prevents human nucleus pulposus cells from IL-1 $\beta$-induced extracellular matrix degradation and apoptosis by inhibiting the NF-kB pathway
}

\author{
LIN LU ${ }^{1}$, JIALANG HU ${ }^{1}$, QIPENG WU ${ }^{1}$, YING AN ${ }^{1}$, WEI CUI ${ }^{1}$, JUNWEN WANG ${ }^{1}$ and ZHEWEI YE ${ }^{2}$ \\ ${ }^{1}$ Department of Orthopaedics, Wuhan Fourth Hospital; Puai Hospital, Tongji Medical College, \\ Huazhong University of Science and Technology, Wuhan, Hubei 430033; ${ }^{2}$ Department of Orthopaedics, Union Hospital, \\ Tongji Medical College, Huazhong University of Science and Technology, Wuhan, Hubei 430022, P.R. China
}

Received October 17, 2018; Accepted February 8, 2019

DOI: 10.3892/ijmm.2019.4105

\begin{abstract}
Intervertebral disc degeneration (IDD) is widely considered to be one of the main causes of lower back pain, which is a chronic progressive disease closely related to inflammation, nucleus pulposus (NP) cell apoptosis and extracellular matrix (ECM) degradation. Berberine (BBR) is an alkaloid compound with an anti-inflammatory effect and has been reported to exert therapeutic action in several inflammatory diseases, including osteoarthritis. Therefore, it was hypothesized that BBR may have a therapeutic effect on IDD through inhibition of the inflammatory response. The aim of the present study was to evaluate the influence of BBR on IDD in interleukin (IL)-1 $\beta$-treated human NP cells in vitro. The results showed that BBR attenuated the upregulation of ECM-catabolic factors [matrix metalloproteinase (MMP)-3, MMP-13, a disintegrin and metalloproteinase with thrombospondin motif (ADAMTS)-4 and ADAMTS-5], and the downregulation of ECM-anabolic factors (type II collagen and aggrecan) following stimulation of the human NP cells with IL-1 $\beta$. Treatment with BBR also protected human NP cells from IL-1 $\beta$-induced apoptosis, as determined by western blotting and flow cytometry. Mechanistically, the IL-1 $\beta$-stimulated degradation of I $\mathrm{KB} \alpha$, and the phosphorylation and translocation of nuclear factor
\end{abstract}

Correspondence to: Dr Junwen Wang, Department of Orthopaedics, Wuhan Fourth Hospital; Puai Hospital, Tongji Medical College, Huazhong University of Science and Technology, 473 Hanzheng Street, Qiaokou, Wuhan, Hubei 430033, P.R. China

E-mail:1ledu2017@163.com

Dr Zhewei Ye, Department of Orthopaedics, Union Hospital, Tongji Medical College, Huazhong University of Science and Technology, 1277 Jiefang Avenue, Wuhan, Hubei 430022, P.R. China

E-mail: 1289097683@qq.com

Key words: intervertebral disc degeneration, berberine, inflammation, nuclear factor- $\mathrm{\kappa B}$, extracellular matrix degradation, apoptosis
(NF)- $\mathrm{B}$ p65 were found to be attenuated by BBR, indicating that NF- $\kappa B$ pathway activation was suppressed by BBR in the IL-1 $\beta$-treated human NP cells. The results of the experiments revealed a therapeutic potential of BBR for the prevention or treatment of IDD.

\section{Introduction}

Lower back pain (LBP) is a common musculoskeletal disorder that severely affects the quality of life of human beings and imposes a substantial economic burden on society (1). Intervertebral disc (IVD) degeneration (IDD) has been shown to be the major contributor to LBP (2). IVD, composed of a central nucleus pulposus (NP) surrounded by annulus fibrosus and cartilaginous endplates, is an avascular organ. In healthy IVD, the hydrated NP, which is mainly composed of NP cells and is rich in the extracellular matrix (ECM), including type II collagen and aggrecan, is important in the physiological function of the disc in distributing the mechanical load acting on the spine, and in multi-axial flexibility $(3,4)$. During the progression of IDD, the increased levels of pro-inflammatory cytokines, elevated cell apoptosis, increased production of ECM-catabolic proteinases, including matrix metalloproteinases (MMPs) and a disintegrin and metalloproteinase with thrombospondin motifs (ADAMTS), and decreased synthesis of type II collagen and aggrecan have been observed within the NP tissue (5-7). These cellular and molecular changes lead to disruption of the physiological structure and function of IVD and to spinal instability, which are the main triggers of LBP (8).

The excessive production of proinflammatory molecules secreted by NP cells, including tumor necrosis factor (TNF)- $\alpha$, interleukin (IL)-1 $\alpha$, IL-1 $\beta$, IL-2 and IL- 6 , has been demonstrated to serve critical roles in the initiation and development of IDD $(5,9)$. Among the above-mentioned inflammatory cytokines, IL-1 $\beta$ is widely considered to be the predominant cytokine that is expressed at high levels in degenerative IVD tissues and cells and has been shown to be involved in several pathological processes in NP cells, including inflammatory responses, oxidative stress, apoptosis, and an imbalance of ECM synthesis and degradation $(10,11)$. Therefore, inhibiting 
the effect of IL-1 $\beta$ on NP cells may postpone the progression of IDD.

Berberine (BBR) is an isoquinoline alkaloid that is derived from several medicinal herbs, including Rhizoma Coptidis, Cortex Phellodendri, and Mahonia bealei, which have long been used in traditional Chinese medicine. BBR has been reported to possess multiple pharmacological effects, including anti-oxidative, anti-inflammatory and anti-apoptotic effects (12-14). Previous studies have found that BBR has therapeutic effects on musculoskeletal disorders, including rheumatoid arthritis and osteoarthritis, owing to its anti-inflammatory properties $(15,16)$. Zhao et al showed that BBR treatment can protect articular cartilage from degeneration via activating the Akt-p70S6K-S6 signaling pathway in IL-1 $\beta$-stimulated articular chondrocytes and in a rat osteoarthritis model (17). Hu et al reported that BBR decreases glycosaminoglycan release and nitric oxide production in IL-1 $\beta$-stimulated chondrocytes (16). In addition, the administration of BBR was found by Zhou et al to prevent nitric oxide-induced chondrocyte apoptosis and cartilage degeneration in a rat model of osteoarthritis (18). As the morphology and avascular supply of NP cells are similar to those of chondrocytes, and BBR has been reported to inhibit the effects of oxidative stress in rat NP cells (19), it was hypothesized that BBR may prevent the development of IDD by protecting NP cells from IL-1 $\beta$-induced degenerative effects. Therefore, the purpose of the present study was to investigate the influence of BBR on IL-1 $\beta$-induced apoptosis and ECM degradation in human NP cells and to elucidate the underlying molecular mechanism.

\section{Materials and methods}

Patient tissue samples. Between March and October 2017, human lumbar NP tissues were collected from 10 patients (six women and four men; mean age, 24.7 years; age range, 15-42 years) with idiopathic scoliosis who underwent deformity correction surgery with the approval of the Ethics Committee of Tongji Medical College, Huazhong University of Science and Technology (Wuhan, China). Written informed consent was obtained from all participants involved in the study. The degrees of degeneration of the discs of all participants were assessed using the modified Pfirrmann grading system (20) and were classified as grade II.

Human NP cell culture and treatment. Human NP cells were isolated using a method reported previously by Kang et al (21), and were then cultured in DMEM/F12 (Gibco; Thermo Fisher Scientific, Inc., Waltham, MA, USA) containing $15 \%$ of fetal bovine serum (Gibco; Thermo Fisher Scientific, Inc.) and $1 \%$ of a penicillin-streptomycin solution at $37^{\circ} \mathrm{C}$ in a humidified atmosphere containing $5 \% \mathrm{CO}_{2}$. The cells were passaged twice for use in the following experiments. The human NP cells were seeded in a six-well plate at a density of $10^{5}$ cells/well. On reaching 80-90\% confluence, the NP cells were incubated with $25 \mu \mathrm{M}$ BBR for $2 \mathrm{~h}$ prior to IL-1 $\beta$ (10 $\mathrm{ng} / \mathrm{ml})$ treatment for $24 \mathrm{~h}$ at $37^{\circ} \mathrm{C}$. The NP cells were harvested for subsequent experiments. All experiments were conducted in triplicate.

Cell viability analysis. Cell viability was evaluated using a Cell Counting Kit-8 (CCK-8, Dojindo Molecular Technologies, Inc.,
Kumamoto, Japan). Briefly, the human NP cells were seeded in a 96-well plate $\left(5 \times 10^{3}\right.$ cells per well) and cultured as described above, followed by treatment with various concentrations of $\operatorname{BBR}(5,10,15,20$ or $25 \mu \mathrm{M})$ or IL-1 $\beta(10 \mathrm{ng} / \mathrm{ml})$ for $24 \mathrm{~h}$ at $37^{\circ} \mathrm{C}$. Subsequently, $10 \mu \mathrm{l}$ of the CCK- 8 dye was added into each well, followed by incubation at $37^{\circ} \mathrm{C}$ for $2 \mathrm{~h}$. The optical density was measured at $450 \mathrm{~nm}$ on a microplate reader (Leica Microsystems GmbH, Wetzlar, Germany).

RNA extraction and reverse transcription-quantitative polymerase chain reaction $(R T-q P C R)$ analysis. Total RNA was isolated from the human NP cells with TRIzol reagent (Invitrogen; Thermo Fisher Scientific, Inc.). The mRNA expression levels of type II collagen, aggrecan, MMP-3, MMP-13, ADAMTS-4 and ADAMTS-5 were quantified by RT-qPCR analysis on a 7500 Real-Time PCR system (Applied Biosystems; Thermo Fisher Scientific, Inc.) under the cycling conditions recommended by the manufacturer. qPCR was performed using a SYBR Prime Script ${ }^{\mathrm{TM}}$ RT-qPCR kit (Takara Biotechnology Co., Ltd., Dalian, China). The reaction conditions were as follows: $95^{\circ} \mathrm{C}$ for $10 \mathrm{~min}$, followed by 40 cycles at $95^{\circ} \mathrm{C}$ for $30 \mathrm{sec}$ and $60^{\circ} \mathrm{C}$ for $30 \mathrm{sec}$. The gene expression levels were normalized to that of $\beta$-actin. Relative expression levels were analyzed using the $2^{-\Delta \Delta \mathrm{Cq}}$ method (22). The primer sequences used for RT-qPCR analysis were as follows: Type II collagen, forward 5'-AGAACTGGTGGAGCAGCAAGA-3' and reverse 5'-AGCAGGCGTAGGAAGGTCAT-3'; aggrecan, forward 5'-TGAGCGGCAGCACTTTGAC-3' and reverse 5'-TGAGTACAGGAGGCTTGAGG-3'; MMP-3, forward 5'-TTCCTTGGATTGGAGGTGAC-3' and reverse 5'-AGC CTGGAGAATGTGAGTGG-3'; MMP-13, forward 5'-CCC AACCCTAAACATCCAA-3' and reverse 5'-AAACAGCTC CGCATCAACC-3'; ADAMTS-4, forward 5'-ACCCAAGCA TCCGCAATC-3' and reverse 5'-TGCCCACATCAGCCA TAC-3'; ADAMTS-5, forward 5'-GACAGTTCAAAGCCA AAGACC-3' and reverse 5'-TTTCCTTCGTGGCAGAGT-3'; $\beta$-actin, forward 5'-AGCGAGCATCCCCCAAAGTT-3' and reverse 5'-GGGCACGAAGGCTCATCATT-3'.

Western blotting. The western blotting procedure was performed to analyze protein levels. The treated human NP cells were lysed with RIPA lysis buffer, and protein concentration was measured using the BCA Protein Assay kit (Beyotime Institute of Technology, Haimen, China). The nuclear and cytoplasmic proteins were isolated with the Nuclear/Cytosolic Fractionation kit (BioVision, Inc., Mountain View, CA, USA). The concentration was measured using a BCA protein assay. A total of $40 \mu \mathrm{g}$ protein per lane was separated by sodium dodecyl sulphate polyacrylamide gel electrophoresis on a $12 \%$ gel and transferred onto a polyvinylidene fluoride membrane (EMD Millipore, Billerica, MA, USA). Following blocking with 5\% non-fat milk in TBST, the membranes were incubated with primary antibodies against type II collagen (sc-7764; $142 \mathrm{kDa}$; Santa Cruz Biotechnology, Inc., Dallas, TX, USA; 1:8,000), aggrecan (ab3778; 50-60 kDa; Abcam, Cambridge, UK; 1:100), MMP-3 (14351; 60 kDa; Cell Signaling Technology, Inc., Danvers, MA, USA; 1:1,000), MMP-13 (ab39012; $54 \mathrm{kDa}$; Abcam; 1:4,000), ADAMTS-4 (ab185722; $90 \mathrm{kDa}$; Abcam; 1:1,000), ADAMTS-5 (ab41037; 73 kDa; Abcam; 

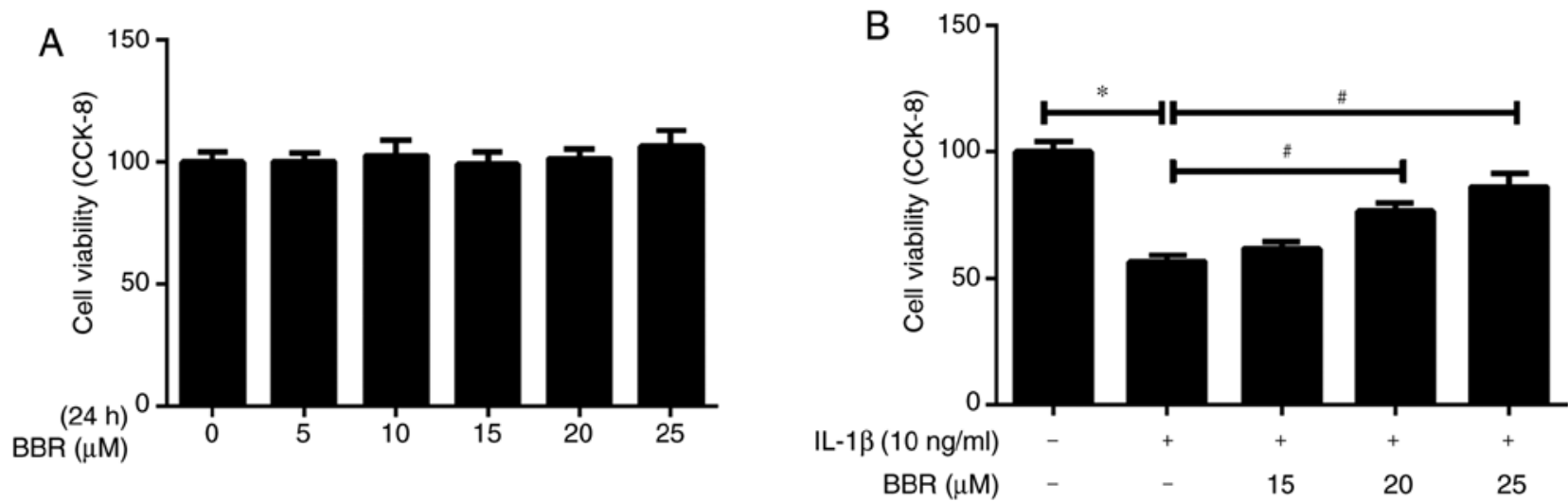

Figure 1. Protective effect of BBR on human NP cell viability. (A) Influence of the indicated concentrations of BBR on the viability of NP cells at 24 h, as measured by a CCK-8 assay. (B) CCK- 8 results of BBR-pretreated NP cells stimulated by IL-1 $\beta$. Data are presented as the mean \pm standard deviation. ${ }^{*}<0.05$, vs. control group; ${ }^{~} \mathrm{P}<0.05$, vs. IL-1 $\beta$ group. NP, nucleus pulposus; BBR, berberine; CCK-8, Cell Counting Kit-8; IL-1 $\beta$, interleukin-1 $\beta$.

1:200), B-cell lymphoma 1 (Bcl-2; ab32124; 26 kDa; Abcam; 1:1,000), Bcl-2-associated X protein (Bax; ab32503; $20 \mathrm{kDa}$; Abcam; 1:1,000), cleaved caspase3 (9664; $17 \mathrm{kDa}$; Cell Signaling Technology, Inc.; 1:1,000), nuclear factor (NF)- $\kappa \mathrm{B}$ p65 (ab16502; 60 kDa; Abcam; 1:500), phosphorylated p65 (p-p65; ab86299; 60 kDa; Abcam; 1:1,000), inhibitor of $\kappa \mathrm{B} \alpha(\mathrm{I} \kappa \mathrm{B} \alpha$; (9242; $39 \mathrm{kDa}$; Cell Signaling Technology, Inc.; 1:1,000), $\beta$-actin (ab8227; $42 \mathrm{kDa}$; Abcam; 1:2,000) and lamin B1 (ab16048; $66 \mathrm{kDa}$; Abcam; 1:1,000) overnight at $4^{\circ} \mathrm{C}$, followed by incubation with the respective secondary antibodies (BA1054; Boster Biological Technology, Pleasanton, CA, USA, 1:5,000) at room temperature for $1 \mathrm{~h}$. The protein bands were detected using an enhanced chemiluminescence system and analyzed quantitatively using BandScan software (version 4.30; BioMarin Pharmaceutical Inc., UK). $\beta$-actin and lamin B1 served as loading controls.

Flow cytometry. Following treatment with the different agents, the human NP cells were harvested, washed with cold PBS, and stained using the Annexin V-FITC Apoptosis Detection kit (Beyotime Institute of Biotechnology, Haimen, China). The fluorescence of the cells was determined immediately by flow cytometry (BD Biosciences, Franklin Lakes, NJ, USA). The apoptotic rate was calculated as the sum of the percentages of early (Annexin $\mathrm{V}^{+} / \mathrm{PI}^{-}$) and late $\left(\right.$Annexin $\mathrm{V}^{+} / \mathrm{PI}^{+}$) apoptotic cells.

Statistical analysis. The results are expressed as the mean \pm standard deviation. Statistical analyses were performed using SPSS v.18.0 software (SPSS, Inc., Chicago, IL, USA). Differences between groups were evaluated by one-way analysis of variance followed by the Tukey test. $\mathrm{P}<0.05$ was considered to indicate a statistically significant difference.

\section{Results}

Cell viability of human NP cells following treatment with $B B R$. The present study evaluated the effect of BBR on the viability of human NP cells at various concentrations $(5,10$, 15,20 or $25 \mu \mathrm{M}$ ) for $24 \mathrm{~h}$ using the CCK- 8 assay. The results indicated that BBR was not significantly toxic to human
NP cells at concentrations ranging between 5 and $25 \mu \mathrm{M}$ (Fig. 1A). In addition, BBR was administered to IL-1 $\beta$-treated human NP cells at various concentrations $(15,20$ or $25 \mu \mathrm{M})$. Pretreatment with BBR had a significant protective effect against IL-1 $\beta$-induced cell apoptosis, particularly at $25 \mu \mathrm{M}$ (Fig. 1B). Therefore, $25 \mu \mathrm{M}$ BBR was selected for subsequent experiments.

Inhibitory effects of BBR on IL-1 $\beta$-induced ECM degradation by human NP cells. To determine whether BBR affects IL-1 $\beta$-induced ECM degradation by human NP cells, the present study assessed the mRNA and protein expression levels of the main components of the NP ECM, including type II collagen and aggrecan, and major NP ECM catabolic proteinases MMP-3, MMP-13, ADAMTS-4 and ADAMTS-5 by RT-qPCR and western blot analyses, respectively. The results revealed that IL-1 $\beta$ treatment markedly decreased the mRNA expression levels of type II collagen and aggrecan, whereas pretreatment with BBR attenuated the downregulation induced by IL-1 $\beta$ (Fig. $2 \mathrm{~A}$ and B). It was also found that the mRNA expression levels of MMP-3, MMP-13, ADAMTS-4 and ADAMTS-5 in human NP cells were significantly increased following stimulation with IL-1 $\beta$ (Fig. 2C-F). However, BBR pretreatment resulted in a statistically significant attenuation of IL-1 $\beta$-induced upregulation of these ECM catabolic proteinases (Fig. 2C-F). The same results were observed for protein expression levels (Fig. 2G-M)

$B B R$ protects human NP cells from IL-1 $\beta$-induced cell apoptosis. As NP cell apoptosis is important in the progression of IDD, the present study investigated the influence of BBR on IL-1 $\beta$-induced human NP cell apoptosis. Flow cytometric analysis revealed that IL- $1 \beta$ increased the rate of apoptosis compared with that in the untreated control (Fig. 3A and B). When the human NP cells were cotreated with BBR and IL-1 $\beta$, the results showed a significant decrease in the rate of apoptosis (Fig. 3A and B). Furthermore, the expression levels of apoptosis-related proteins (Bcl-2, Bax and cleaved caspase3) were assessed. The protein levels of Bax (pro-apoptotic) and cleaved caspase 3 (pro-apoptotic) were increased and the protein level of Bcl-2 (anti-apoptotic) was decreased in the 

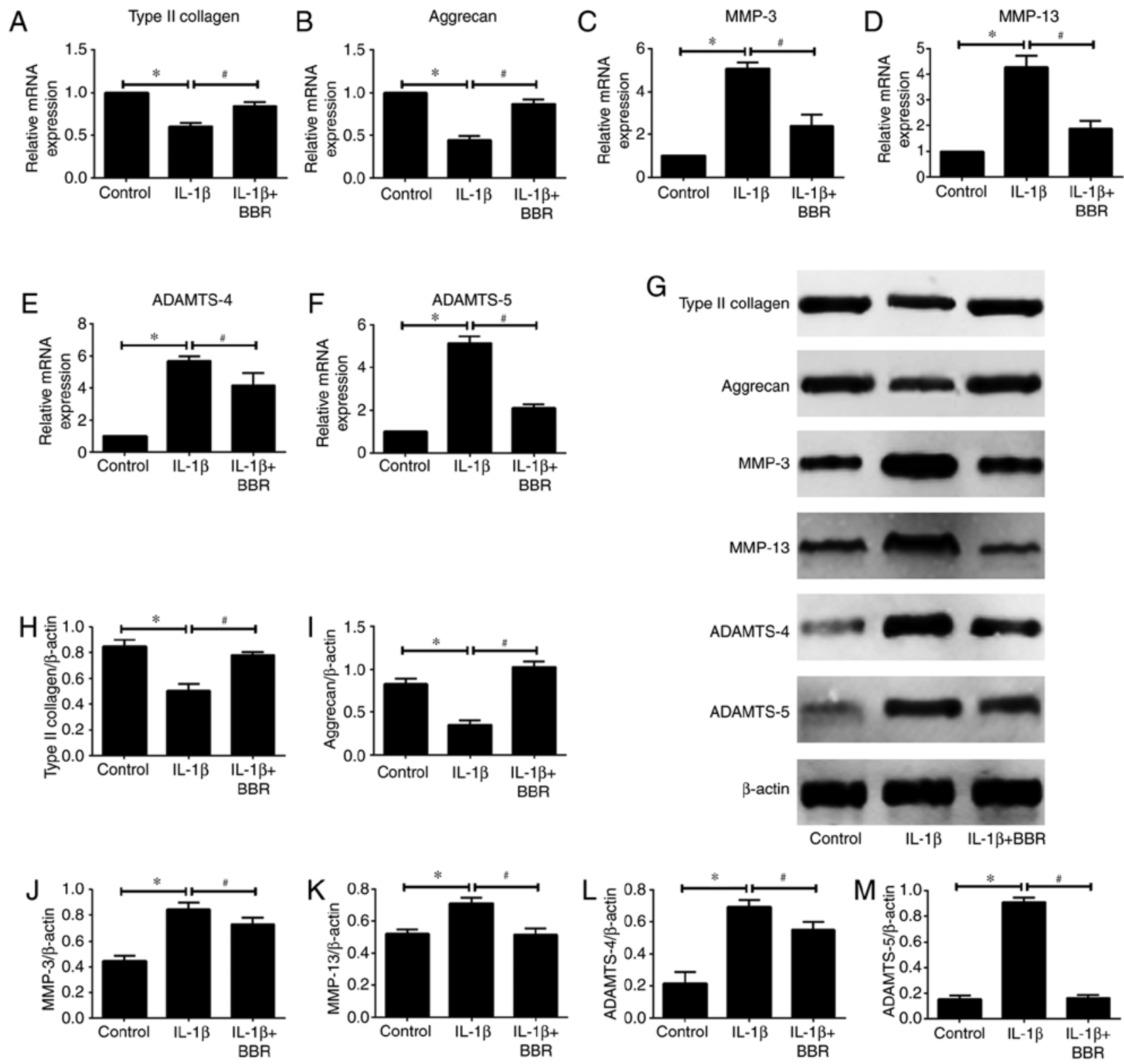

Figure 2. Effects of BBR on IL-1 $\beta$-induced extracellular matrix degradation by human NP cells. Following treatment with BBR $(25 \mu \mathrm{M})$ for $2 \mathrm{~h}, \mathrm{NP}$ cells were stimulated with IL-1 $13(10 \mathrm{ng} / \mathrm{ml})$ for $24 \mathrm{~h}$ and harvested for RT-qPCR and western blot analyses. mRNA expression of (A) type II collagen, (B) aggrecan, (C) MMP-3, (D) MMP-13, (E) ADAMTS-4 and (F) ADAMTS-5, as analyzed by RT-qPCR analysis. (G) Western blot analysis of the protein levels of (H) type II collagen, (I) aggrecan, (J) MMP-3, (K) MMP-13, (L) ADAMTS-4 and (M) ADAMTS-5. $\beta$-actin served as an internal control. Data are presented as the mean \pm standard deviation. "P<0.05, vs. control group; ${ }^{\prime} \mathrm{P}<0.05$, vs. IL-1 $\beta$ group. NP, nucleus pulposus; BBR, berberine; IL-1 $\beta$, interleukin-1 $\beta$; MMP, matrix metalloproteinase; ADAMTS, a disintegrin and metalloproteinase with thrombospondin motifs; RT-qPCR, reverse transcription-quantitative polymerase chain reaction.

IL-1 $\beta$-treated human NP cells (Fig. 3C-G). These trends were reversed by BBR pretreatment (Fig. 3C-G).

Suppressive effects of BBR on the IL-1 $\beta$-induced activation of $N F-\kappa B$ in human NP cells. The NF-кB pathway has been found to be aberrantly activated in IDD and has been demonstrated to be an important mediator of the IL-1 $\beta$-induced degenerative effects in human NP cells. Therefore, to further examine the underlying mechanism of BBR-induced anti-degenerative effects, the present study determined whether BBR regulates the NF- $\kappa$ B pathway in IL-1 $\beta$-treated human NP cells. The extent of NF- $\mathrm{KB}$ p65 phosphorylation and the intracellular distribution of p65 were first evaluated by western blotting. Compared with the untreated group, a higher ratio p-p65/p65 and nuclear translocation of p65 from the cytoplasm were observed following IL-1 $\beta$ stimulation (Fig. 4A-C), indicating activation of the NF- $\mathrm{KB}$ signaling pathway in the IL-1 $\beta$-treated human NP cells. However, pretreatment with BBR markedly suppressed this IL-1 $\beta$-induced phosphorylation and translocation of p65 (Fig. 4A-C). IкB $\alpha$ is an inhibitor protein for $\mathrm{p} 65$, the phosphorylation of $\mathrm{I} \kappa \mathrm{B} \alpha$ and its subsequent degradation induce the translocation of p65 to the nucleus. Therefore, the level of cytoplasmic I $\mathrm{I} B \alpha$ was analyzed by western blotting in human NP cells. IL-1 $\beta$ treatment induced the degradation of cytoplasmic $\mathrm{I} \kappa \mathrm{B} \alpha$, whereas this effect was attenuated by BBR pretreatment (Fig. 4D).

\section{Discussion}

LBP affects $80 \%$ of the global population, and IDD is widely recognized as the main cause of LBP (2). The prevention or 

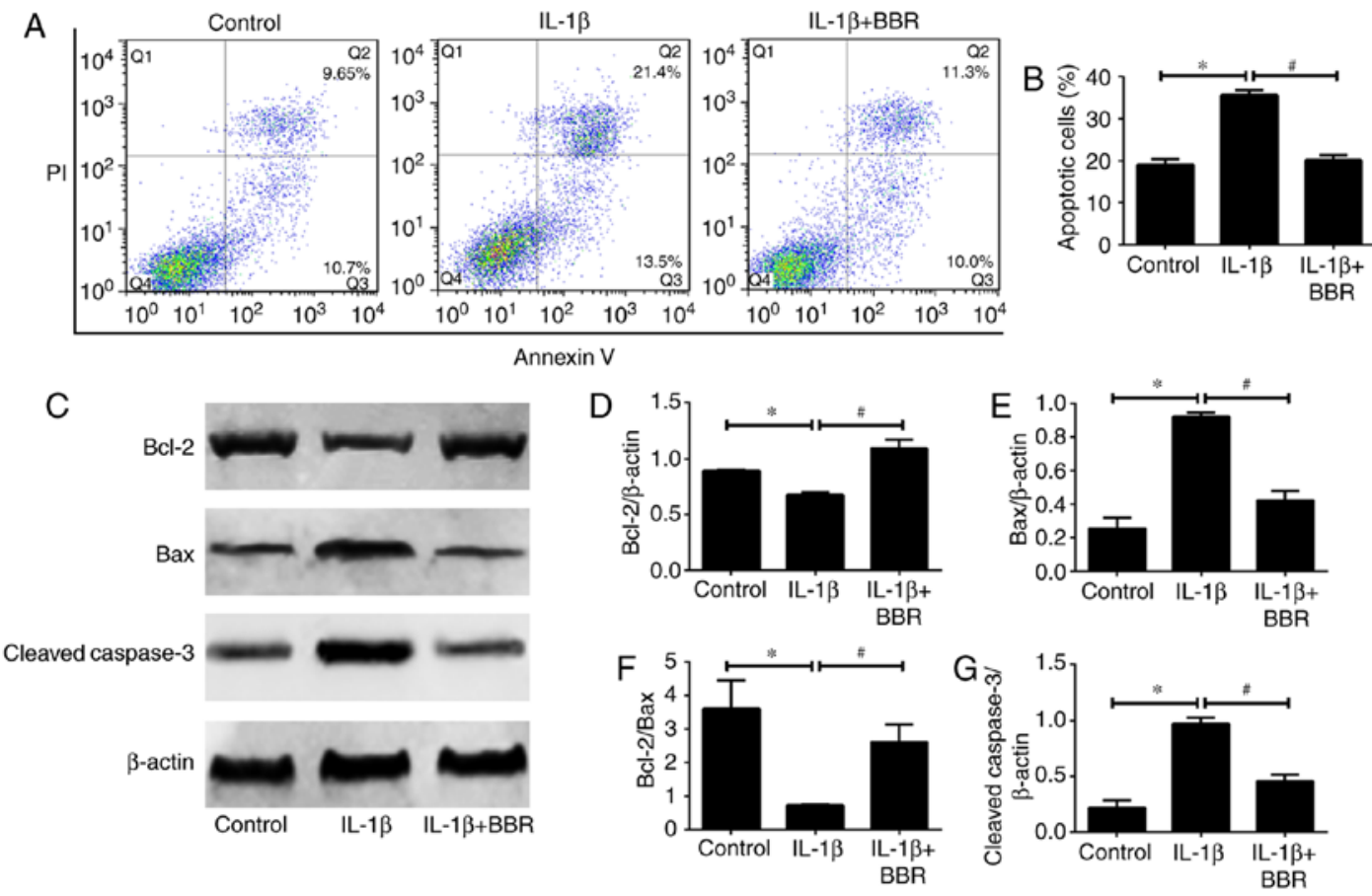

Figure 3. Impact of BBR on IL-1 $\beta$-induced human NP cells apoptosis. Following treatment with BBR ( $25 \mu \mathrm{M})$ for 2 h, NP cells were stimulated with IL-1 $\beta$ $(10 \mathrm{ng} / \mathrm{ml})$ for $24 \mathrm{~h}$ and harvested for flow cytometry and western blotting. (A) Flow cytometry was used to determine the (B) rates of apoptosis of human NP cells. (C) Western blot analysis and quantification of protein levels of (D) Bcl-2, (E) Bax, (F) Bcl-2/Bax and (G) cleaved caspase 3. $\beta$-actin served as an internal control. Data are presented as the mean \pm standard deviation. ${ }^{*} \mathrm{P}<0.05$, vs. control group; ${ }^{\#} \mathrm{P}<0.05$, vs. IL- $1 \beta$ group. NP, nucleus pulposus; BBR, berberine; IL-1 $\beta$, interleukin-1 $\beta$; Bcl-2, B-cell lymphoma 2; Bax, Bcl-2-associated X protein.

A
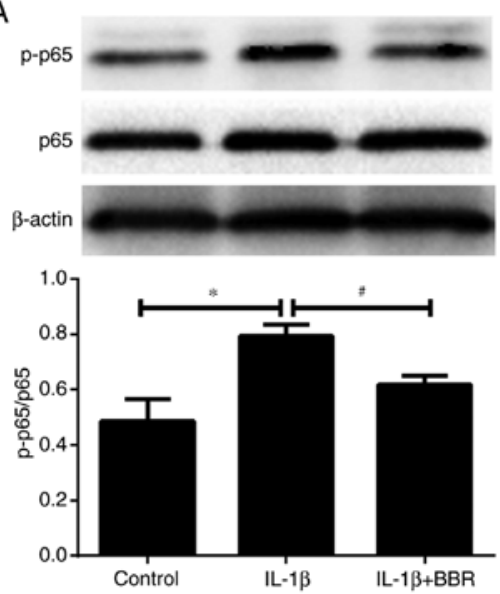

C

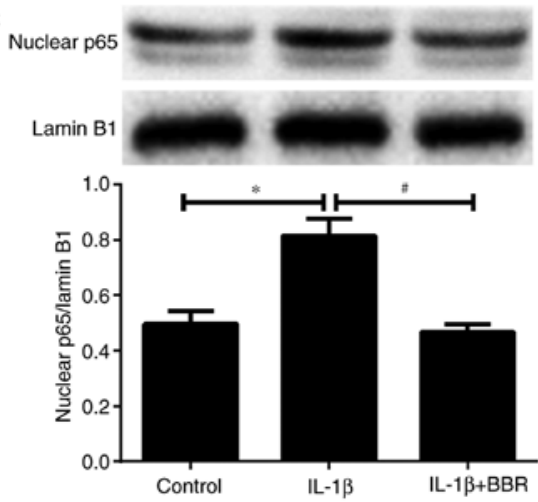

B

Cytoplasmic p65

$\beta$-actin

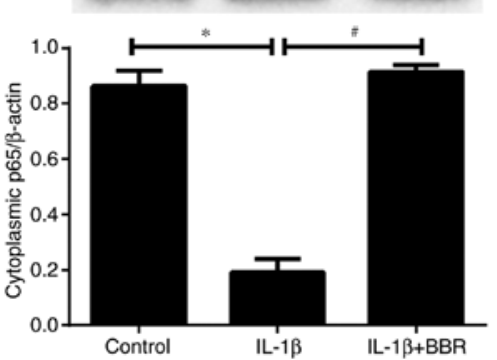

D

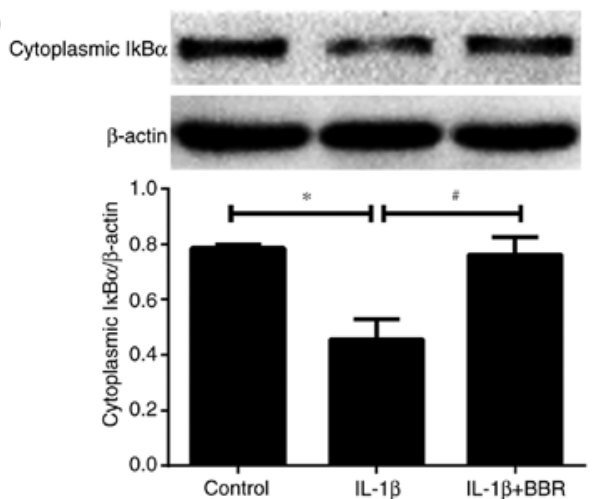

Figure 4. Effects of BBR on IL-1 $\beta$-induced NF- $\kappa$ B pathway activity in human NP cells. (A) Total protein levels of NF- $\kappa$ B p65 and its phosphorylated form were ana-

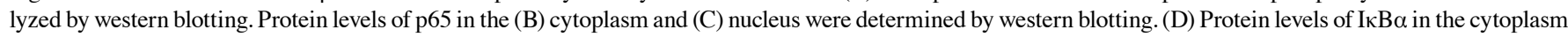
were evaluated by western blotting. $\beta$-actin or lamin B1 was used as an internal control. Data are presented as the mean \pm standard deviation. ${ }^{*} \mathrm{P}<0.05$, vs. control group; ${ }^{~} \mathrm{P}<0.05$, vs. IL-1 $\beta$ group. NP, nucleus pulposus; BBR, berberine; IL-1 $\beta$, interleukin-1 $\beta$; NF- $\kappa \mathrm{B}$, nuclear factor- $\kappa \mathrm{B}$; I $\kappa \mathrm{B} \alpha$, inhibitor of $\kappa \mathrm{B} \alpha$; $\mathrm{p}-$, phosphorylated. 


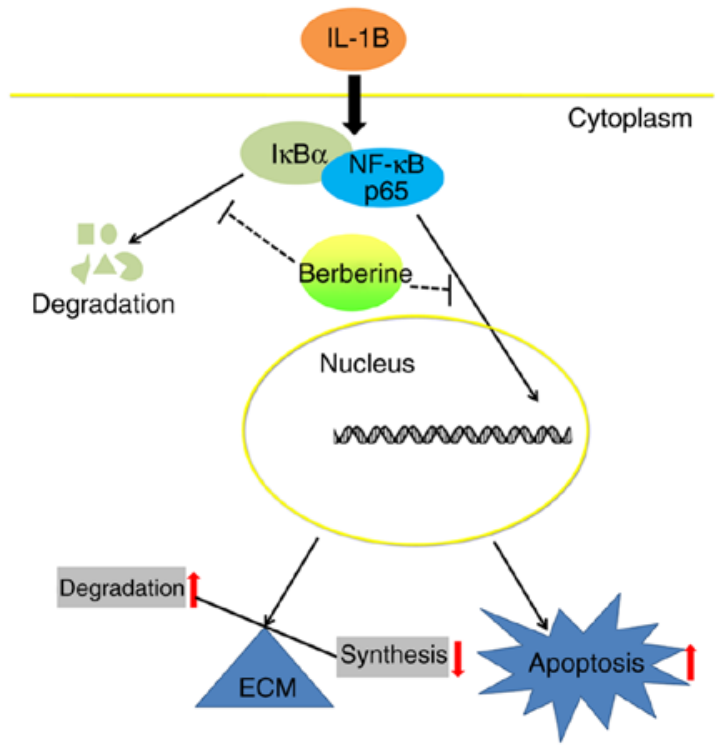

Figure 5. Schematic model of the signaling mechanisms underlying the inhibitory effect of BBR on ECM catabolism and apoptosis in IL-1 $\beta$-stimulated human NP cells. NP, nucleus pulposus; BBR, berberine; IL-1 $\beta$, interleukin-1 $\beta$; NF- $\kappa B$, nuclear factor- $\kappa \mathrm{B}$; I $\kappa \mathrm{B} \alpha$, inhibitor of $\kappa \mathrm{B} \alpha$; ECM, extracellular matrix.

reversal of IDD is a potential treatment of LBP; however, the pathological basis and mechanisms underlying IDD remain to be fully elucidated. Current evidence suggests that the excessive inflammatory response, an increase in the proportion of apoptotic NP cells, and the imbalance between anabolism and catabolism of NP ECM are closely associated with the development of $\operatorname{IDD}(7,8,23)$; therefore, targeting these pathological processes is a novel strategy for the treatment of IDD. Medical treatments of LBP caused by IDD mainly include conservative approaches and, rarely, surgical procedures. Conservative approaches, including non-steroidal anti-inflammatory drugs, are prescribed to inhibit inflammation and to relieve the symptom of back pain temporarily; however, the widespread and long-term use of non-steroidal anti-inflammatory drugs results in considerable adverse effects (24). Surgical procedures, including spine fusion and discectomy (25), which cannot preserve the function of IVD, imposes a financial burden on the patient and is not an optimal choice. Therefore, investigating novel drug treatments that can promote endogenous repair of degenerative IVD and prevent IDD development is crucial, particularly for early-stage IDD. In previous years, plant-derived compounds have attracted the attention of those investigating IDD treatment owing to their anti-inflammatory effects and few adverse effects $(10,26)$. BBR, an isoquinoline alkaloid extracted from Coptidis Rhizoma and Cortex Phellodendri, has been shown to have potent therapeutic potential against inflammatory diseases $(12,27)$. The present study provides the first evidence, to the best of our knowledge, that BBR has pharmacological inhibitory effects on ECM degradation and apoptosis in IL-1 $\beta$-stimulated human NP cells. The results also showed that BBR inhibits the IL-1 $\beta$-induced activation of the NF- $\mathrm{kB}$ pathway in human NP cells (Fig. 5).

IL-1 $\beta$ is a major risk factor of IDD, with potent proinflammatory activity involving stimulation of the production of multiple proinflammatory mediators (28). It has been demonstrated that IL-1 $\beta$ is involved in multiple pathological processes related to the development of IDD $(5,10,29)$. The progressive degradation of the NP ECM, which mainly consists of type II collagen and aggrecan (performing a vital function in the maintenance of the physiological function of IVD), is the most prominent feature of IDD. MMPs and ADAMTSs are the major ECM-degrading enzymes in IDD, and their functions in IDD have been investigated extensively (30). MMP-3 and MMP-13 have been identified as the main collagenases that lead to degradation of type II collagen in NP, whereas ADAMTS-4 and ADAMTS-5 are the primary aggrecanases due to their potent and specific activity in cleaving aggrecan. The expression of all these enzymes, which are often regarded as the catabolic markers of IDD, has been found to be upregulated in degenerative NP (31). The inhibition of MMPs and ADAMTSs has a therapeutic effect on IDD in vitro and in vivo. There is substantial evidence that, in NP cells, IL-1 $\beta$ can promote the production of ECM-degrading enzymes that degrade type II collagen and aggrecan in NP ECM $(5,10)$. In the present study, the results showed that IL-1 $\beta$ treatment resulted in the upregulation of MMP-3, MMP-13, ADAMTS- 4 and ADAMTS-5 and the downregulation of type II collagen and aggrecan in human NP cells, as expected, and that BBR reversed these changes induced by IL-1 1 . These results suggest that BBR has the potential to control the progression of IDD by restoring the balance between ECM anabolism and catabolism in human NP cells.

NP cells are important for maintaining the structural integrity of IVD by generating the molecular components of ECM. Recently, an apoptosis-associated decrease in the NP cell number was proposed as an important mechanism underlying the development of IDD $(32,33)$. Therefore, treatment of IDD not only depends on the NP ECM homeostasis but also on inhibiting the apoptosis of NP cells. Several studies have demonstrated that IL- $1 \beta$ is an important regulator of the apoptosis of NP cells $(34,35)$. The majority of rabbit NP cells were shown to undergo apoptosis following IL-1 $\beta$ stimulation and exhibit morphological changes related to apoptosis, whereas a combined treatment with insulin-like growth factor 1 and IL-1 $\beta$ significantly reduced IL- $1 \beta$-mediated apoptosis (35). Shen et al (34) reported that IL-1 $\beta$ induces the mitochondrial pathway in NP cells by increasing the expression ratio Bax/Bcl-2 and by releasing cytochrome $c$ from the mitochondria to the cytoplasm, subsequently activating downstream caspases 9 and 3 to complete the apoptotic process. In addition, Chen et al (19) found that BBR may mitigate oxidative-stress-induced apoptosis through the mitochondrial pathway. The results of flow cytometric analysis in the present study revealed that BBR effectively prevented IL-1 $\beta$-induced apoptosis. The data also indicated that BBR attenuated the downregulation of $\mathrm{Bcl}-2$ and the upregulation of Bax and cleaved caspase 3 at the protein level in IL-1 $\beta$-treated human NP cells. Taken together, these results suggest that BBR protects human NP cells from IL-1 $\beta$-induced apoptosis.

Various intracellular signaling pathways are activated in response to inflammatory stimulation associated with IDD, thereby mediating the increase in the production of a downstream effector that is closely involved in the progression of IDD (36). As one of the most critical intracellular signaling proteins, NF- $\mathrm{kB}$ can regulate the expression of 
genes associated with ECM degradation and cell apoptosis in IL-1 $\beta$-treated human NP cells $(21,37)$. Inhibiting the activation of NF- $\kappa \mathrm{B}$ is regarded as a potential therapeutic strategy against IDD. Under normal conditions, $N F-\kappa B$ is located in the cytoplasm bound to an inhibitory protein, $\mathrm{I} \kappa \mathrm{B}$, which prevents $N F-\kappa B$ from entering the nucleus. Upon stimulation by IL- $1 \beta$, the I $\kappa \mathrm{B}$ protein is phosphorylated and degraded, resulting in the translocation of $\mathrm{NF}-\kappa \mathrm{B}$ from the cytoplasm to the nucleus. Subsequently, NF- $\mathrm{B}$ facilitates gene transcription by binding to specific sequences in the promoter region of NF- $\kappa \mathrm{B}$-responsive genes, which upregulate the production of catabolic enzymes, inflammatory mediators and cytokines $(5,10)$. To further elucidate the molecular mechanism underlying the inhibitory effect of BBR on ECM degradation and apoptosis in IL- $1 \beta$-treated NP cells, the present study assessed the influence of BBR on the IL-1 $\beta$-induced activation of NF- $\kappa \mathrm{B}$ in human NP cells. The results revealed that BBR significantly inhibited the IL- $1 \beta$-induced upregulation of the phosphorylation of $\mathrm{NF}-\kappa \mathrm{B}$ p65 and its nuclear translocation in human NP cells. In addition, the IL- $1 \beta$-induced decrease in the level of cytoplasmic I $\kappa \mathrm{B} \alpha$ was reversed by BBR, indicating that treatment with BBR inhibited the degradation of $\mathrm{I} \kappa \mathrm{B} \alpha$, thereby maintaining $\mathrm{NF}-\kappa \mathrm{B}$ in an inactive state. Taken together, these results suggest that the therapeutic action of BBR on IDD may be mediated by suppression of the $\mathrm{NF}-\kappa \mathrm{B}$ signaling pathway.

However, as human NP cells cultured in vitro and human NP cells in vivo may respond differently to BBR treatment, whether BBR is effective as a IDD treatment in vivo remains to be fully elucidated. Therefore, animal experiments that can truly mimic IDD pathogenesis are urgently required to confirm the protective effect of BBR against IDD and to advance current understanding of the molecular mechanisms underlying this effect.

In conclusion, the results of the present study suggest that BBR exerts potent anti-ECM catabolic and anti-apoptotic actions by inhibiting the IL- $1 \beta$-induced activation of NF- $\kappa$ B in human NP cells, indicating that BBR may be validated as an effective therapeutic agent for IDD in the future.

\section{Acknowledgements}

Not applicable.

\section{Funding}

No funding was received.

\section{Availability of data and materials}

The datasets used and/or analyzed during the current study are available from the corresponding author on reasonable request.

\section{Authors' contributions}

JW and ZY designed the study. LL wrote the manuscript. LL, $\mathrm{JH}$, and QW conducted the experiments. LL, JH, QW, YA, and WC collected the data. JW, ZY, and LL analyzed the data. JW and $\mathrm{ZY}$ reviewed and revised the manuscript. All authors read and approved the manuscript and agree to be accountable for all aspects of the research in ensuring that the accuracy or integrity of any part of the work are appropriately investigated and resolved.

\section{Ethics approval and consent to participate}

The present study was approved by the Ethics Committee of Tongji Medical College, Huazhong University of Science and Technology (Wuhan, China).

\section{Patient consent for publication}

Not applicable.

\section{Competing interests}

The authors declare that they have no competing interests.

\section{References}

1. Martin BI, Deyo RA, Mirza SK, Turner JA, Comstock BA, Hollingworth W and Sullivan SD: Expenditures and health status among adults with back and neck problems. JAMA 299: 656-664, 2008.

2. Luoma K, Riihimaki H, Luukkonen R, Raininko R, Viikari-Juntura $\mathrm{E}$ and Lamminen A: Low back pain in relation to lumbar disc degeneration. Spine (Phila Pa 1976) 25: 487-492, 2000.

3. Pattappa G, Li Z, Peroglio M, Wismer N, Alini M and Grad S: Diversity of intervertebral disc cells: Phenotype and function. J Anat 221: 480-496, 2012.

4. Sakai D and Grad S: Advancing the cellular and molecular therapy for intervertebral disc disease. Adv Drug Deliv Rev 84: 159-171, 2015.

5. Yang W, Yu XH, Wang C, He WS, Zhang SJ, Yan YG, Zhang J, Xiang YX and Wang WJ: Interleukin- $1 \beta$ in intervertebral disk degeneration. Clin Chim Acta 450: 262-272, 2015.

6. Wu B, Meng C, Wang H, Jia C and Zhao Y: Changes of proteoglycan and collagen II of the adjacent intervertebral disc in the cervical instability models. Biomed Pharmacother 84: 754-758, 2016.

7. Li Y, Li K, Han X, Mao C, Zhang K, Zhao T and Zhao J: The imbalance between TIMP3 and matrix-degrading enzymes plays an important role in intervertebral disc degeneration. Biochem Biophys Res Commun 469: 507-514, 2016.

8. Freemont AJ: The cellular pathobiology of the degenerate intervertebral disc and discogenic back pain. Rheumatology (Oxford) 48: 5-10, 2009.

9. Yamamoto J, Maeno K, Takada T, Kakutani K, Yurube T, Zhang Z, Hirata H, Kurakawa T, Sakai D, Mochida J, et al: Fas ligand plays an important role for the production of pro-inflammatory cytokines in intervertebral disc nucleus pulposus cells. J Orthop Res 31: 608-615, 2013.

10. Chen J, Xuan J, Gu YT, Shi KS, Xie JJ, Chen JX, Zheng ZM, Chen Y, Chen XB, Wu YS, et al: Celastrol reduces IL-1 $\beta$ induced matrix catabolism, oxidative stress and inflammation in human nucleus pulposus cells and attenuates rat intervertebral disc degeneration in vivo. Biomed Pharmacother 91: 208-219, 2017.

11. Risbud MV and Shapiro IM: Role of cytokines in intervertebral disc degeneration: Pain and disc content. Nat Rev Rheumatol 10: 44-56, 2014.

12. Wang Z, Chen Z, Yang S, Wang Y, Huang Z, Gao J, Tu S and Rao Z: Berberine ameliorates collagen-induced arthritis in rats associated with anti-inflammatory and anti-angiogenic effects. Inflammation 37: 1789-1798, 2014.

13. Li Z, Geng YN, Jiang JD and Kong WJ: Antioxidant and anti-inflammatory activities of berberine in the treatment of diabetes mellitus. Evid Based Complement Alternat Med 2014: 289264, 2014.

14. Liang Y, Huang M, Jiang X, Liu Q, Chang X and Guo Y: The neuroprotective effects of Berberine against amyloid $\beta$-protein-induced apoptosis in primary cultured hippocampal neurons via mitochondria-related caspase pathway. Neurosci Lett 655: 46-53, 2017. 
15. Wang X, He X, Zhang CF, Guo CR, Wang CZ and Yuan CS Anti-arthritic effect of berberine on adjuvant-induced rheumatoid arthritis in rats. Biomed Pharmacother 89: 887-893, 2017.

16. Hu PF, Chen WP, Tang JL, Bao JP and Wu LD: Protective effects of berberine in an experimental rat osteoarthritis model Phytother Res 25: 878-885, 2011.

17. Zhao H, Zhang T, Xia C, Shi L, Wang S, Zheng X, Hu T and Zhang B: Berberine ameliorates cartilage degeneration in interleukin-1 $\beta$-stimulated rat chondrocytes and in a rat model of osteoarthritis via Akt signalling. J Cell Mol Med 18: 283-292, 2014.

18. Zhou Y, Liu SQ, Yu L, He B, Wu SH, Zhao Q, Xia SQ and Mei HJ: Berberine prevents nitric oxide-induced rat chondrocyte apoptosis and cartilage degeneration in a rat osteoarthritis model via AMPK and p38 MAPK signaling. Apoptosis 20: 1187-1199, 2015.

19. Chen Y, Zheng Z, Wang J, Tang C, Khor S, Chen J, Chen X, Zhang Z, Tang Q, Wang C, et al: Berberine suppresses apoptosis and extracellular matrix (ECM) degradation in nucleus pulposus cells and ameliorates disc degeneration in a rodent model. Int J Biol Sci 14: 682-692, 2018

20. Pfirrmann CW, Metzdorf A, Zanetti M, Hodler J and Boos N: Magnetic resonance classification of lumbar intervertebral disc degeneration. Spine (Phila Pa 1976) 26: 1873-1878, 2001.

21. Kang L, Hu J, Weng Y, Jia J and Zhang Y: Sirtuin 6 prevents matrix degradation through inhibition of the NF- $\kappa \mathrm{B}$ pathway in intervertebral disc degeneration. Exp Cell Res 352: 322-332, 2017.

22. Livak KJ and Schmittgen TD: Analysis of relative gene expression data using real-time quantitative PCR and the 2(-Delta Delta C(T)) Method. Methods 25: 402-408, 2001.

23. Purmessur D, Walter BA, Roughley PJ, Laudier DM, Hecht AC and Iatridis $\mathrm{J}$ : A role for $\mathrm{TNF} \alpha$ in intervertebral disc degeneration: A non-recoverable catabolic shift. Biochem Biophys Res Commun 433: 151-156, 2013

24. Roelofs PD, Deyo RA, Koes BW, Scholten RJ and van Tulder MW: Nonsteroidal anti-inflammatory drugs for low back pain: An updated Cochrane review. Spine (Phila Pa 1976) 33: 1766-1774, 2008.

25. Phillips FM, Reuben $\mathbf{J}$ and Wetzel FT: Intervertebral disc degeneration adjacent to a lumbar fusion. An experimental rabbit model. J Bone Joint Surg Br 84: 289-294, 2002.

26. Zhang $\mathrm{B}, \mathrm{Xu} \mathrm{L}$, Zhuo $\mathrm{N}$ and Shen J: Resveratrol protect against mitochondrial dysfunction through autophagy activation in human nucleus pulposus cells. Biochem Biophys Res Commun 493: 373-381, 2017.

27. Chandirasegaran G,Elanchezhiyan C, Ghosh K and Sethupathy S: Berberine chloride ameliorates oxidative stress, inflammation and apoptosis in the pancreas of Streptozotocin induced diabetic rats. Biomed Pharmacother 95: 175-185, 2017.
28. Jimbo K, Park JS, Yokosuka K, Sato K and Nagata K: Positive feedback loop of interleukin-1beta upregulating production of inflammatory mediators in human intervertebral disc cells in vitro. J Neurosurg Spine 2: 589-595, 2005.

29. Johnson ZI, Schoepflin ZR, Choi H, Shapiro IM and Risbud MV: Disc in flames: Roles of TNF- $\alpha$ and IL-1 $\beta$ in intervertebral disc degeneration. Eur Cells Mater 30: 104-116; discussion 116-117, 2015.

30. Wang WJ, Yu XH, Wang C, Yang W, He WS, Zhang SJ, Yan YG and Zhang J: MMPs and ADAMTSs in intervertebral disc degeneration. Clin Chim Acta 448: 238-246, 2015.

31. Kang L, Yang C, Yin H, Zhao K, Liu W, Hua W, Wang K, Song Y, Tu J, Li S, et al: MicroRNA-15b silencing inhibits IL-1 $\beta$-induced extracellular matrix degradation by targeting SMAD3 in human nucleus pulposus cells. Biotechnol Lett 39: 623-632, 2017.

32. Han Y, Li X, Yan M, Yang M, Wang S, Pan J, Li L and Tan J: Oxidative damage induces apoptosis and promotes calcification in disc cartilage endplate cell through $\mathrm{ROS} / \mathrm{MAPK} / \mathrm{NF}-\kappa \mathrm{B}$ pathway: Implications for disc degeneration. Biochem Biophys Res Commun: Mar 23, 2017 (Epub ahead of print).

33. Cheng X, Zhang L, Zhang K, Zhang G, Hu Y, Sun X, Zhao C, Li H, Li YM and Zhao J: Circular RNA VMA21 protects against intervertebral disc degeneration through targeting miR-200c and X linked inhibitor-of-apoptosis protein. Ann Rheum Dis 77: 770-779, 2018

34. Shen J, Xu S, Zhou H, Liu H, Jiang W, Hao J and Hu Z: IL-1 $\beta$ induces apoptosis and autophagy via mitochondria pathway in human degenerative nucleus pulposus cells. Sci Rep 7: 41067, 2017.

35. Zhang CC, Zhou JS, Hu JG, Wang X, Zhou XS, Sun BA, Shao C and Lin Q: Effects of IGF-1 on IL-1 $\beta$-induced apoptosis in rabbit nucleus pulposus cells in vitro. Mol Med Rep 7: 441-444, 2013.

36. Wuertz K, Vo N, Kletsas D and Boos N: Inflammatory and catabolic signalling in intervertebral discs: The roles of NF- $\kappa \mathrm{B}$ and MAP kinases. Eur Cell Mater 23: 103-119; discussion 119-120, 2012.

37. Li Z, Wang X, Pan H, Yang H, Li X, Zhang K, Wang H, Zheng Z, Liu $\mathrm{H}$ and Wang J: Resistin promotes CCL4 expression through toll-like receptor- 4 and activation of the p38-MAPK and NF- $\mathrm{kB}$ signaling pathways: Implications for intervertebral disc degeneration. Osteoarthritis Cartilage 25: 341-350, 2017.

This work is licensed under a Creative Commons Attribution-NonCommercial-NoDerivatives 4.0 International (CC BY-NC-ND 4.0) License. 